\title{
Preface: Physical Fitness and Functional Performance in People with Musculoskeletal and Neurologic Disorders and Challenges to Rehabilitation in Middle-Income Countries
}

Physical fitness in the general population is determined largely by multiple modifiable factors including nutrition, physical activity, attitude toward health, and individual genetics. Similarly, following injury or disease, outcome is largely dependent on modifiable contextual factors that are both personal and environmental. Hence, for health promotion and rehabilitation, fitness and functional outcomes rely on modifiable variables that are influenced by socioeconomic and cultural influences.

Healthcare providers who face twin challenges of maximizing fitness and function know that it is critical to improve overall health in heavily resource-constrained societies. Identification of simple, naturally evolved models will help to equip health-care agencies with effective strategies to bring about positive changes in overall health. Such models can be globally extended across national boundaries to low- and mediumincome societies.

In a developing country such as India, a huge gap exists between access to health care and affordability in urban and rural health sectors. India's expenditures on health comprise just $>1 \%$ of its gross domestic product (GDP), ${ }^{1}$ compared to $9.2 \%$ of GDP per capita disbursement on healthcare in Europe. ${ }^{2}$ In addition, the disproportionate public/ private split of healthcare results in greater reliance on private care, posing a huge challenge for maintaining overall health in the nearly 1.4 billion Indian population. ${ }^{3}$

Mahatma Gandhi Mission School of Physiotherapy, Navi Mumbai, a constituent unit of Mahatma Gandhi Mission Institute of Health Sciences in Maharashtra, India, is a leading self-funded private institute that received seed funding in 2008 and strives to emerge as a center of excellence in physiotherapy education, research, and clinical care. The Institute's mandate is to offer physiotherapeutic services to urban and rural communities to prevent disease and promote health and rehabilitation. Its long-term vision is to train graduate students through masters and doctoral degree programs to meet or exceed international levels of practice in physiotherapy and innovative research.

The Institute offers services to more than 12,000 patients annually at two tertiarycare hospitals of the Mahatma Gandhi Mission Trust, with three nongovernmental organizations in Navi Mumbai. In addition, physiotherapy is provided to rural populations in Navi Mumbai, Raigad, and Thane districts. We serve a population of $>1.1$ million in catchment areas. The sustainable model of patient care offers specialized cardiopulmonary, neurology, and musculoskeletal physiotherapy at intensive-care units to rural communities, extending from antenatal care to neonatal, pediatric, adult, and geriatric populations. 
Our research activities focus on health promotion, rehabilitation, and discovering affordable, sustainable, evidence-based solutions with collaborative interdisciplinary efforts. A team of ten faculty members and 26 graduate students work in a research-intensive environment. Joined by 17 investigators from nearby academic institutions, we maintain an environment of scientific inquiry through internally and externally funded research. A large patient population with diverse pathologies has resulted in a researchintensive environment.

Major areas of investigation include biomechanical exploration of movement in daily living, sports, and dance at Mahatma Gandhi Mission Center of Human Movement Science, Navi Mumbai. Need-based research projects focus on clinical rehabilitation of neuro-musculo-skeletal disorders, health promotion, and prevention of noncommunicable diseases at a tertiary-care level in the community. In parallel, the Center works to design healthcare technology for early detection, evaluation, and rehabilitation. It is devoted to the development of robust, affordable, technology solutions for health problems. We have filed two patents jointly with the Indian Institute of Technology Bombay for devices designed for objective, early detection of diabetic neuropathy.

On the basis of our research during the past 10 years, we are pleased to present in this special issue ten articles exploring physical fitness and functional outcome in people with metabolic and neuro-musculo-skeletal disorders, with a view to rehabilitate. We focus on disorders such as spine and knee pain, metabolic disorders such as type 2 diabetes mellitus (T2DM), and neurological disorders including spinal cord injury, cerebral palsy, and Parkinson's disease (PD). Our research examines the challenges encountered by our investigators, especially during rehabilitation of those with these pathologies.

Spine pain is a major musculoskeletal burden occurring at a high rate of $42 \%$ in India. ${ }^{4}$ We observed that $\sim 43.5 \%$ of dancers practicing classical Indian dance $(50 \%$ were Bharatanatyam dancers) suffer from low back pain. ${ }^{5}$ Mullerpatan et al. report novel findings on gait kinematics in Bharatanatyam dancers presenting with low back pain. ${ }^{6}$ The work is pioneering and could lead to targeted regimens for prevention and rehabilitation following dance-related injuries. ${ }^{6}$

Knee pain, the second-most prevalent musculoskeletal disorder in India, occurs in $\sim 28 \%$ of individuals. ${ }^{7}$ Our efforts to promote health by planning rehabilitation programs for prevention and management and reducing the huge burdens of noncommunicable diseases such as diabetes and osteoarthritis led to exploratory studies to understand levels of fitness and influence of squat exposure on knee pain. Physical therapist Bela Agarwal and her team investigated varying squat exposure on knee pain and function among people with osteoarthritis. ${ }^{8}$ Their research elucidates the benefits of knee motion, muscle strength, and balance in those with greater squat exposure, implying potential benefits of deep-squat activity performed in moderation to maintain knee range, muscle strength, and balance, despite knee osteoarthritis. ${ }^{8}$

Shruti Nair et al. explore health-related fitness among people with T2DM. ${ }^{9}$ Notable reductions in cardiorespiratory endurance; muscle flexibility, strength, and endurance; and balance compared to those in healthy adults suggest a strong need to increase awareness regarding benefits of physical activity, regular fitness assessments, and training in routine clinical practice. ${ }^{9}$ 
Long-term rehabilitation of people with spinal cord injuries revealed that patients discontinue walking with assistive devices and revert to the use of wheelchairs even after rigorous rehabilitation, ultimately losing the benefits of the weight-bearing physical activity that is offered by walking. Hence, Naryanan et al. explore energy expenditure during various walking techniques with assistive devices, which are considered to be essential for training people with spinal cord injury. ${ }^{10}$ For individuals with dorsolumbar spinal cord injury, the authors recommend four-point gait using crutches for therapeutic value during indoor training and outdoor walking. ${ }^{10}$

The limited information currently available on sports participation among people with spinal cord injury in low- to middle-income countries prompted Pal to study this topic along with fellow investigators who have expertise in musculoskeletal, cardiopulmonary, and sports physiotherapy. ${ }^{11}$ Research objectives included exploring barriers to participation and performance and bringing these to the attention of policy makers in government and the private sector, thereby introducing sustained efforts for improvement. In people with spinal cord injury, sports participation was found to be $60 \%$ in spinal cord rehabilitation facilities of Mumbai. Strong family support, financial security, and institutional support in the form of motivation and training were top facilitators. Barriers faced during participation in sports included difficulty in reaching the sports ground, lack of disabled-friendly toilets, and accommodation. ${ }^{11}$

At our institute, care for pediatric cerebral palsy patients ranges from instrumented three-dimensional gait analysis to supplemental evaluation for precise clinical decision making in surgical and nonsurgical management of long-term rehabilitation. Sheti and colleagues studied the gait deviation index for children with severe gait impairment to gain insight and assist clinicians in monitoring the effects of surgical and nonsurgical interventions in pediatric cerebral palsy patients. ${ }^{12}$ Our observations in focused schools and rehabilitation centers reveal that routine care lacks integration of high-flexion postures such as squats, which are required for functioning in a traditional lifestyle. Therefore, we studied motor patterns adopted by healthy children to attain upright posture from a squat during a period of $12 \mathrm{mo}$. Or results can be integrated into therapy. ${ }^{13}$

Clinical evaluation by Shetty et al. of lower-extremity muscle strength and endurance in ambulatory children led to targeted pediatric therapy programs addressing varying levels of gross motor function. ${ }^{14}$ Continued efforts regarding rehabilitation of children with cerebral palsy and intellectual disabilities, along with caregiver support, have evolved to offer comprehensive rehabilitative care to improve physical function, psychological and cognitive performance, education, and sports participation, by engaging specialists within the targeted area of expertise. ${ }^{14}$ Low levels of competitive sports participation among children with mild intellectual disabilities have led to the identification of challenges that may be faced during sports participation. Shetty et al. reported a low level $(47 \%)$ of sports participation among these children at the competitive level, with only $13 \%$ participating at the national level. Findings warrant serious attention to plan interventions to improve sports participation and performance of children with mild intellectual disabilities. ${ }^{15}$ 
During the course of rehabilitation of individuals with PD, we explored hand-dexterity function and revealed that subjects demonstrate notably less manual and fine dexterity compared to age- and gender-matched healthy subjects. We studied manual and fine dexterity using a nine-hole peg test and the Minnesota Manual Dexterity Test. Our findings will provide clinicians with reference values for time taken to perform in normal healthy subjects and those with PD, assisting in training manual and fine dexterity for hand rehabilitation of patients with PD. ${ }^{16}$

In this special issue of Critical Reviews ${ }^{\mathrm{TM}}$ in Physical and Rehabilitation Medicine, our multidisciplinary teams consisted of national and international investigators who present research conducted at Mahatma Gandhi School of Physiotherapy, Navi Mumbai and collaborating academic centers. Researchers include physiotherapists, surgeons, human movement scientists, biomedical engineers, and a professional Indian classical dancer. Interdisciplinary research on physical and rehabilitation medicine with leading national institutes such as the Indian Institute of Technology, Bombay; Indian Institute of Technology, Madras; and Kaivalyadhama Shreeman Madhava Yoga Mandira Samiti, Lonavla, India have helped to establish Mahatma Gandhi Mission School of Physiotherapy as a leading center for both patient care and research in physical and rehabilitation medicine in India. Our collaboration with international facilities at Cardiff University, United Kingdom; University of Sydney, Australia; and World Spine Care Organization have resulted in publications in leading journals. ${ }^{17,18}$ We believe that our research transcends national boundaries and will benefit patients across widespread global communities.

\section{ACKNOWLEDGMENTS}

The research reported in this invited special issue is conducted via generous grants from the Department of Biotechnology, Indian Government, International Society of Biomechanics, and Mahatma Gandhi Mission Institute of Health Sciences, Navi Mumbai. We thank all subjects and volunteers who participated in our studies.

\section{Guest Editor:}

Dr. Rajani Mullerpatan, MSc PT, PhD

Professor-Director

Mahatma Gandhi Mission School of Physiotherapy, Navi Mumbai, India

MGM School of Physiotherapy

Sector 1, MGM Campus

Kamothe, Panvel, Navi Mumbai, Maharashtra 410206

Rajani Mullerpatan, MSc, PT, PhD, is a qualified physiotherapist at Seth GS Medical College and King Edward Memorial Hospital, Mumbai, India. She specialized in musculoskeletal physiotherapy at Lokmanya Tilak Memorial Medical College and Lokmanya Tilak Memorial General Hospital, Mumbai. Later, she obtained her PhD from Cardiff 
University in Wales. Dr. Mullerpatan has been awarded the Dr. PN Berry Scholarship from the High Commission of India, London, UK and an honorary research fellowship from Cardiff University. Currently, Dr. Mullerpatan advises the Research Cell Society of Indian Physiotherapists and is an Affiliate Societies Officer for the Executive Council of the International Society of Biomechanics. She has served for 10 years as academic and clinical director of the institute.

\section{REFERENCES}

1. National Health Profile data, Ministry of Health and Family Welfare, Government of India, 2018. Available from: http://www.cbhidghs.nic.in/WriteReadData/1892s/Before\%20Chapter1.pdf.

2. Sengupta A. The private health sector in India is burgeoning, but at the cost of public health care. BMJ. 2005 Nov 19; 331(7526):1157-8.

3. World Health Organization. Country-by-country profiles on the national health situation. Data and evidence, Europe 2016. Available from: http://www.euro.who.int/en/data-and-evidence/news/ news/2016/05/country-by-country-profiles-on-national-health-situation-relaunched.

4. Nag A, Vyas H, Nag P. Occupational health scenario of Indian informal sector. Indus Health. $2016 \mathrm{Jul}$ 31;54(4):377-85.

5. Nair SP, Kotian S, Hiller C, Mullerpatan R. Survey of musculoskeletal disorders among Indian dancers in Mumbai and Mangalore. J Dance Med Sci. 2018 Jun 15;22(2):67-74.

6. Mullerpatan R, Bharnuke J, Hiller C. Gait kinematics in Bharatanatyam dancers with and without low back pain. Crit Rev Phys Rehab Med. 2019;31(1):1-10.

7. Pal CP, Singh P, Chaturvedi S, Pruthi KK, Vij A. Epidemiology of knee osteoarthritis in India and related factors. Indian J Orthopaed. 2016;50(5):518.

8. Agarwal B, Advani M, van Deursen R, Mullerpatan R. Influence of varying squat exposure on knee pain and function among people with knee osteoarthritis. Crit Rev Phys Rehab Med. 2019;31(1):11-22.

9. Nair S, Agarwal B, Chatla J, Mullerpatan R. Health-related physical fitness of people with type 2 diabetes mellitus. Crit Rev Phys Rehab Med. 2019;31(1):23-33.

10. Naryanan G, Bose M, Parab S, Agarwal B, Mullerpatan R. Energy expenditure using three different walking techniques in individuals with spinal cord Injury. Crit Rev Phys Rehab Med. 2019;31(1):35-41.

11. Amberkar O, Agarwal B, Singh Y, Shete R, Mullerpatan R. Level of sports participation and performance among people with spinal cord injury. Crit Rev Phys Rehab Med. 2019;31(1):43-51.

12. Shetty T, Johari A, Ganesan S, Mullerpatan R. Exploration of gait deviation index in children with cerebral palsy with severe gait impairment. Crit Rev Phys Rehab Med. 2019;31(1):53-62.

13. Mullerpatan R, Thanawala M, Agarwal B, Ganesan S. Development of deep-squat milestone in typically developing children. Crit Rev Phys Rehab Med. 2019;31(1):63-73.

14. Shetty T, Parab S, Ganesan S, Mullerpatan R. Lower extremity muscle strength and endurance in ambulatory children with cerebral palsy. Crit Rev Phys Rehab Med. 2019;31(1):75-84.

15. Iyer P, Shetty T, Ganesan S, Nair S, Rao N, Mullerpatan R. Exploration of sports participation in children with mild intellectual disability. Crit Rev Phys Rehab Med. 2019;31(1):85-92.

16. Shah T, Shetty M, Bose M, Mullerpatan R. A study of fine and manual hand dexterity among people with Parkinson's disease. Crit Rev Phys Rehab Med. 2019;31(1):93-100.

17. Nordin M, Randhawa K, Torres P, Yu H, Haldeman S, Côté P, Torres C, Modic M, Mullerpatan R, Cedraschi C, Chou R. The Global Spine Care Initiative: A systematic review for the assessment of spinerelated complaints in populations with limited resources and in low-and middle-income communities. Eur Spine J. 2018 Sep 1;27(6):816-27.

18. Johnson CD, Haldeman S, Chou R, Nordin M, Green BN, Côté P, Hurwitz EL, Kopansky-Giles D, Acaroğlu E, Cedraschi C, Ameis A. The Global Spine Care Initiative: Model of care and implementation. Eur Spine J. 2018 Sep 1;27(6):925-45. 
